\title{
A Review on Shape Memory Alloy Structures
}

\author{
Basavaraj S. Balapgol, Sudhakar A. Kulkarni, Kamal M. Bajoria \\ Department of Civil Engineering, Indian Institute of Technology Bombay, Powai, Mumbai 400076, India
}

(Received 6 June 2003; revised 27 February 2004; accepted 3 March 2004)

\begin{abstract}
This paper presents a literature review on smart material NiTi shape memory alloys and their structural applications. Shape memory alloys are capable of recovering large strains as a result of martensitic transformation. The NiTi shape memory alloy is widely used because of its large memory strain, superelastic behaviour and greater thermal stability. The review includes the behaviours and the characteristics of the NiTi shape memory alloys, and also covers, in brief, structural applications and limitations.
\end{abstract}

\section{INTRODUCTION}

The study of smart materials and structures has received considerable attention in recent years. The advantages of incorporating these special types of materials into a structure is that the sensing and the actuating mechanism becomes part of the structure so that one can monitor structural integrity and take corrective actions. There are a number of materials that have the capability to be used as a sensor or an actuator or both. Piezoelectric materials, magnetostrictive materials, electrostrictive materials, shape memory alloys, and electrorheological fluids are widely known examples. Among these, shape memory alloys (SMAs) are metals and exhibit two very unique properties, shape memory effect and superelasticity.

Ölander ${ }^{1}$ discovered the pseudoelastic behaviour of the Au-Cd alloy in 1932. Greninger and Mooradian ${ }^{2}$ observed the formation and disappearance of a martensitic phase by decreasing and increasing the temperature of a $\mathrm{Cu}-\mathrm{Zn}$ alloy. Chang and $\operatorname{Read}^{3}$ reported the basic phenomenon of the memory effect governed by the thermoelastic behaviour of the martensite phase. Buehler and Wang ${ }^{4}$ at the U.S. Naval Ordinance Laboratory discovered the shape memory effect in an equiatomic alloy of nickel and titanium, which can be considered a breakthrough in the field of shape memory materials. This alloy was named as nitinol (Nickel-Titanium Naval Ordinance Laboratory), after the Naval Ordinance Laboratory now known as the Naval Surface Weapons Center. Since that time, intensive investigations have been made to elucidate the mechanics of its basic behaviour. According to Castleman et al. ${ }^{5}$ the first efforts to exploit the potential of NiTi as an implant material were made by Johnson and Alicandri in 1960s. In the last few years, several publications on numerical modelling and experimental studies have demonstrated the efficiency of shape memory alloys in structural applications. ${ }^{6}$

The term "shape memory alloy" (SMA) is applied to that group of metallic materials that demonstrate the ability to return to some previously defined shape or size when subjected to appropriate thermal and/or mechanical changes. The SMAs undergo a crystalline phase change when heated or cooled above the ambient temperature besides alloy composition and heat treatment, and this phase change is accompanied by a change in the elastic modulus and the critical stress. SMA materials such as, nickel titanium (NiTi), Cu-Zn-Al, Cu-Al$\mathrm{Ni}$, some ferrous alloys, shape memory ceramics and shape memory polymers have been widely used for shape and vibration control. Applications have occurred in various engineering fields viz. aeronautical, civil, mechanical. The transformation of the NiTi to an intermediate phase brings about an excellent shape memory property with small hysteresis. Also because of its better thermal stability, the NiTi has become more popular than other SMAs in engineering fields.

When the NiTi SMA is cold or below its transformation temperature, it has a very low critical stress and can be deformed quite easily into any new shape. ${ }^{7}$ However, when the material is heated above its transformation temperature, it undergoes a change in crystal structure, which causes it to return to its original shape. If the NiTi encounters resistance during its transformation, then extremely large forces will be generated. The NiTi also recovers large strain (up to 8\%), making it unique in applications, such as, actuators for active stiffness, active and passive shape control, vibration control and the flutter control of structures. ${ }^{8}$

\section{CHARACTERISTICS OF SHAPE MEMORY ALLOYS}

The characteristics of shape memory materials are marked by the low critical stress in the martensitic phase and the low modulus of elasticity at low temperatures. However these characteristics are completely changed to high critical stress, high modulus of elasticity and increased damping capacity at elevated temperatures. Cross et al. ${ }^{9}$ presented experimental results of variation of the modulus of elasticity as shown in Fig. 1 and the variation of $2 \%$ critical stress (see Fig. 2). The nickel content in the NiTi alloy varies from 49 to $57 \%$ by weight. ${ }^{10}$ The SMA exhibits the austenite phase, the martensite phase, and the mixture of both. In the austenite phase, the material has a high modulus of elasticity and a high critical stress. When it is cooled, it transforms to the martensite phase where it has a low modulus of elasticity and a low critical stress. The amount of the material that exists in the martensite phase is measured by the martensitic fraction $(\xi)$ which can vary from zero to one. The temperature at which the material starts transforming from austenite to martensite is called the martensite start temperature $\left(M_{S}\right)$, while the temperature at which it has a martensite fraction of one is called the martensite finish temperature $\left(M_{f}\right)$. When the material is heated, it undergoes a change of phase from martensite to austenite; similarly, there exists an austenite start temperature $\left(A_{s}\right)$ and an austenite finish temperature $\left(A_{f}\right)$ (see Fig. 3). If an alloy is stressed above the $M_{S}$ temperature, an apparent 